\title{
Application of membership function in bounding surface model
}

\author{
Xi-gang Wang ${ }^{1,2, a}$ Ming-fu Fu ${ }^{1, b}$ \\ 1 Center of engineering mechanical experiment, Nanchang University, Nanchang 330031, China \\ 2 College of resources and civil engineering, Liaoning institute of science and technology, Benxi \\ 117004,China \\ afx_wxg@163.com, ${ }^{\mathrm{b}} \mathrm{mffu@nit.edu.cn}$
}

Keywords: fuzzy bounding surface model; the loading-unloading ; membership function; strain accumulation;

Abstract. A three-dimensional cone is established by adopting the critical soil mechanics and the membership function to the bounding surface model to express the dynamic characteristics of the rock-soil materials under loading-unloading; The new axis of the cone expresses the plastic membership function of stress, in which the loading surface corresponds with the membership function; The fuzzy hardening modulus of the loading surface is obtained from that of the boundary surface, the plastic flow criteria are fixed by the membership function; The model can well express the accumulated plastic strain; The model parameters are obtained from dynamic triaxial experiment, and the numerical results obtained from finite element method correspond with the experimental results, proving the model is valuable.

\section{Introduction}

The strain-stress curve of soil is complex under cycled loading; How to express the accumulated plastic strain and the stress-strain hysteresis is a focus problem.

Two methods are used to solve this problem: With first method, the stress-strain hysteresis curves are studied based on the classical elastic-plastic theory; this method can obtain stress-strain relationship one-to-one for each cycle. With the other method, the maximum plastic strain is studied to search its laws with cycles, from which the empirical model can be deduced. The first method has a certain degree of theoretical foundation.

The plastic hardening modulus field theory was proposed by Mroz in 1967. The model includes the border consolidation surface, the initial yield surface and the nested yield surface which express the plastic modulus of the current stress state. Since the plastic modulus changes with stress, it is difficult to obtain numerical results from the model. The nested yield surface is simplified to deduce and obtain the bounding surface model, the model include the bounding surface and the loading surface model, which includes the bounding surface and the loading surface representing a movement yield surface. The plastic modulus of the loading surface is identified by the distance of the stress points between the loading surface and the bounding surface. The keys of the model are the relationship of the stress point and the relationship of the modulus both between the loading surface and the bounding surface.

\section{The membership function}

An elliptic equation is adopted to express the bounding function based on the critical soil mechanics(Figure 1):

$$
F=\frac{q^{2}}{M^{2}}-p * p_{C}+p^{2}=0
$$

where $q-$ generalized shear stress; $p-$ average stress; $p_{c} \longrightarrow$ node between ellipse and $p$ axis; $M-q-p$ slope of the critical state line. 


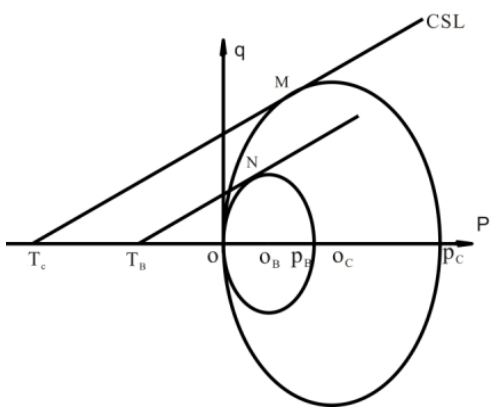

Fig. 1 the bounding surface model

The initial loading surface $F_{O}$ is a point because the plastic strain is generated once the soil is loaded, as shown by point $O$ in Fig.2.The bounding surface $F_{C}$ is the ellipse which passes points $O$ and $P_{C}$, point $O_{C}$ is the center of the ellipse, and $T_{c} M$ is the critical state line corresponding to the bounding surface. Other loading surfaces are expressed by the ellipses that pass point $O$ and ellipse $F_{C}$; For example, ellipse $F_{B}$ which passes points $O$ and $P_{B}$ expresses a loading surface, point $O_{C}$ is the center of the ellipse, and $T_{B} N$ is the critical state line corresponding to the loading surface and is called a fuzzy critical state line.

Axis $O_{C} T_{c}$ is rotated $90^{\circ}$ outward base center $O_{C}$, with which the critical state line and the fuzzy critical state lines are rotated outward $90^{\circ}$. The new three-dimensional cone is established by vertex $T_{c}$ and underside is the bounding surface and the new axis $O_{C} T_{C}$ (Fig.2).

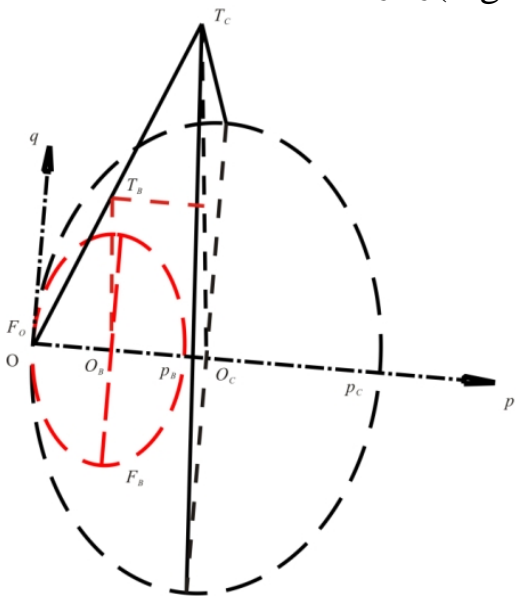

Fig. 2 the cone of membership function

Both $O_{C}$ and $O_{B}$ are the same in the Figures 1 and 2. Nodes $T_{C}$ and $T_{B}$ are obtained when points $O_{C}$ and $O_{B}$ are projected to the new cone, the projection of point $O$ still is $O$; Point $T_{B}$ moves between point $O$ and point $T_{C}$ which express the evolution of the loading surface. Point $T_{B}$ is moving along $O T_{C}$ to $T_{C}$, the loading surface is changed from $F_{B}$ to the bounding surface $F_{C}$; As point $T_{B}$ is moving along $T_{C} O$ to $O$, the loading surface is changed from $F_{B}$ to the initial loading surface $F_{O}$. The coordinates of $T_{B}$ corresponding to the loading surface are adopted to study the evolution of the loading surface (Fig.2).

Axis $O_{C} T_{C}$ expresses the soil plastic membership function, which aims to express the evolution of the loading surface and the difference elastic-plastic degree. Point $T_{C}$ indicates that the loading surface is the bounding surface $F_{C}$ and the membership is equal to 1 . Point $O$ indicate that the loading surface is the initial loading surface $F_{O}$ and the membership is equal to 0 .

$\mu$ is adopted to express the coordinate of $T_{B}$ which is projected to $O_{C} T_{C}$, and then $\mu(p, q) \in[0,1]$. The loading surface change from the initial loading surface $F_{O}$ to the bounding surface $F_{C}$ with $\mu$ changing from 0 to 1 . Coordinate of $T_{B}$ which is projected to $O_{C} T_{C}$ are expressed by the node between axis $\mathrm{p}$ and the loading surface, and thus $\mu$ is defined as follows:

$\mu=P_{B} / P_{C}$

The definition is reasonable and ingenious. $\mu=0$ and $T B$ moves to point $O$ and the loading surface is the initial loading surface when $P_{C}=0 ; \mu=1$ and $T B$ moves to point $T C$ and the loading surface is the bounding surface when $P_{B}=P_{C}$. The loading surface changes from the initial loading surface to the bounding surface when $P_{B}$ changes from $O$ to $P_{C}$. 


\section{Fuzzy elastic-plastic model}

The loading surface changes from the initial surface to the bounding surface with the membership changing from 0 to 1 . The membership is adopted to fuzzy the loading surface based on Eq.(1), and node $p_{c}$ between ellipse and axis $p$ is defined as a fuzzy parameter.

$$
\left\{\begin{array}{c}
\phi^{*}=\frac{q^{4}}{M^{2}}-p * p_{c}^{*}+p^{2}=0 \\
p_{c}^{*}=(1-\mu) * p_{c}
\end{array}\right.
$$

where $\phi^{*}$ equation of fuzzy loading surface; $p_{c}^{*}$ _ node coordinates between fuzzy loading surface and axis $\mathrm{p}$.

The directions of the stress points on the loading surface and are consistent with those of the conjugated stress points on the bounding surface. We suppose obedience to the orthogonal flow rule:

$$
d z^{p}=\left\{\begin{array}{c}
h\left(\frac{\partial \phi^{*}}{\partial \sigma} d \sigma\right) \frac{\partial \phi^{*}}{\partial \sigma} \text { when } \frac{\partial \phi^{*}}{\partial \sigma} \geq 0 \text { and } \mu \geq \mu_{S M} \\
0 \text { when } \frac{\partial \phi^{*}}{\partial \sigma} \leq 0 \text { or when } \frac{\partial \phi^{*}}{\partial \sigma} \geq 0 \text { and } \mu<\mu_{S M}
\end{array}\right.
$$

where $\mu_{S M}$ the membership of memory stress.

Equation (4) indicates that $d \varepsilon^{p}$ is decomposed into a pastic volumetric strain increment $d \varepsilon_{v}^{p}$ and a plastic shear strain increment $d \gamma^{p}$,

$$
\begin{aligned}
& d \varepsilon_{v}^{p}=\left\{\begin{array}{c}
h\left(\frac{\partial \phi^{*}}{\partial p} d p\right) \frac{\partial \phi^{*}}{\partial p} \text { when } \frac{\partial \phi^{*}}{\partial p} \geq 0 \text { and } \mu \geq \mu_{S M} \\
0 \text { when } \frac{\partial \phi^{*}}{\partial p} \leq 0 \text { or when } \frac{\partial \phi^{*}}{\partial p} \geq 0 \text { and } \mu<\mu_{S M}
\end{array}\right. \\
& d \gamma^{p}=\left\{\begin{array}{cc}
h\left(\frac{\partial \phi^{*}}{\partial q} d q\right) \frac{\partial \phi^{*}}{\partial q} \text { when } \frac{\partial \phi^{*}}{\partial q} \geq 0 \text { and } \mu \geq \mu_{S M} \\
0 \text { when } \frac{\partial \phi^{*}}{\partial q} \leq 0 \text { or when } \frac{\partial \phi^{*}}{\partial q} \geq 0 \text { and } \mu<\mu_{S M}
\end{array}\right.
\end{aligned}
$$

where $h$ - fuzzy hardening modulus, which is a function of membership $\mu(\sigma)$ and hardening modulus $H$ of the bounding surface, and $h=h[H, \mu(\sigma)] . H$ is taken as:

$H=p_{i}$

According to consistency condition, Hsatisfies:

$d \emptyset=\frac{\partial \phi}{\partial \sigma_{i j}} d \sigma_{i j}+\frac{\partial \phi}{\partial p_{c}} d H=0$

Supposing $H$ is a function of the plastic strain increment $\varepsilon_{i j} p^{p}$ :

$H=H\left(s_{i j}\right)$

Taking the total differential of $H$ :

$d H=\frac{\partial p_{c}}{\partial s_{i j} p} d \varepsilon_{i j}^{p}$

According to the associated flow rule, the plastic strain increment is expressed as follows:

$d \varepsilon_{i j}{ }^{p}=d \lambda \frac{\partial \phi}{\partial \sigma_{i j}}=H \frac{\partial \phi^{\prime}}{\partial \sigma_{i j}} d \sigma_{i j} \frac{\partial \phi}{\partial \sigma_{i j}}$

$\mathrm{Eq}(15)$ is substituted into Eq (14):

$H=-\frac{1}{\frac{B R}{B H E_{i j} P E \sigma_{i j}}}$

Hardening modulus $h$ of the fuzzy loading surface is determined by $H$ and $\mu$.

$h=h[H, \mu(\sigma)]= \begin{cases}h[H, & 0]=+\infty \\ h[H, & 1]=H\end{cases}$

$h$ is determined based on Eq.(11) as follows:

$h=\left\{1+\tan \left[(1-\mu(\sigma)) * \frac{\pi}{2}\right]\right) * H=\left\{1+\tan \left[(1-\mu(\sigma)) * \frac{\pi}{2}\right]\right\} * p_{c}$

Incremental fuzzy elastic-plastic constitutive equation,Equation (12) is substituted into Eq.(4):

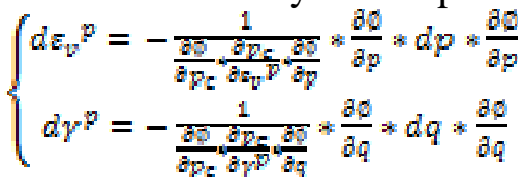

Equation (19) is incremental constitutive equation of the bounding surface, which is obtained by replacing $p_{c}$ with $h$. 


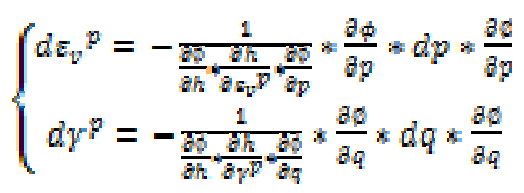

The equation (16) is constitutive equation of the fuzzy bounding surface model. $\mu_{S M}$ is determined by equation (2).

\section{Conclusions}

A new three-dimensional cone is established based on the critical soil mechanics model, and its new axis expresses the plastic membership function of stress; The fuzzy bounding surface model expressing strain accumulation and stress-strain hysteresis of soil is obtained by adopting memory parameter and damage parameter and the membership is used to fix the plastic flow criteria. The parameter of model is obtained from dynamic triaxial test. The numerical results are obtained by further development technology of the finite element method. The numerical results coincide with experimental results, proving that the model is valuable to express strain accumulation and stress-strain hysteresis of soil.

\section{Acknowledgements}

This work was financially supported by the young Start Fund of Liaoning institute of science and technology (0666666),

\section{References}

[1] Mroz,Z..On the description of anisotropic hardening. J. Mech. Phys. Solids, 15(1967),163-175

[2] Krieg R D. A practical two-surface plasticity theory, Journal of Applied Mechanics, ASME, 42(1975),641-646

[3] Dafalias Y F, Popov E P.A model of nonlinearly hardening materials for complex loading, Acta Mechanica,21(1975),173-192.

[4] HUANG Mao-song, LIU Ming, LIU YAN-hua. Anisotropic bounding surface model for saturated soft clay under cyclic loading , Journal of Hydraulic engineering, 40(2009), 188-193.

[5] JIA Peng-fei,KONG Ling-wei,YANG Ai-wu. Strain accumulation model of soils under low-amplitude high-cycle loading, Rock and Soil Mechanics, 34(2013),737-742.

[6] Li D,Selig E T.Cumulative plastic deformation for fine-grained subgrade soils,J.Geotech.Eng.,122(1996),1006-1013.

[7] Chai J C,Miura N,et a1.Traffic-load-induced permanent deformation of road on soft subsoil,Journal of geotechnical and geoenvironmental engineering, 128(2002),907-916.

[8] ZHAO Cong-ming, WAN Quan, JIANG Wei-kang. Numerical analysis and tests for vibration response of a reciprocating compressor,Journal of Vibration and Shock,30( 2011),156-160

[9] GAO, Guang-yun, LI Ning,HE Jun-feng,GAO Meng. Analysis of ground vibration generated by train moving loads on saturated soil,Journal of Vibration and Shock, 30(2011),87-92 